\title{
Influence of torsional stiffness on load sharing coefficient of a power split drive system
}

\author{
Guanghu Jin ${ }^{1, *}$, Wei Ren ${ }^{1}$, and Rupeng Zhu ${ }^{1}$ \\ ${ }^{1}$ Nanjing University of Aeronautics and Astronautics, Nanjing 210016, China
}

\begin{abstract}
A dynamic model of power split transmission system with face gear and cylindrical gear is established. The factors including time-varying mesh stiffness, torsional stiffness, supporting stiffness, and clearance are considered in the model. The influence of the torsional stiffness of compound gear shaft on the load sharing coefficient is analyzed. The results show that the influence of the torsional stiffness of the compound gear shaft is obvious. Because the torsional stiffness of the output gear components is larger and the torsional stiffness of the input gear is smaller, so the input stage's deformation coordination ability is strong. Therefore, with the increase of the torsional stiffness of the compound gear shaft, the load sharing coefficient of the power input stages is improved, but the load sharing coefficient of the split torque stages and power confluence stages is worse. Hence, the torsional stiffness ratio of the transmission shaft should be rationally allocated under the condition that the torsional stiffness of the compound shaft is small.
\end{abstract}

\section{Introduction}

In the transmission system of the helicopter main reducer, compared with the planetary gear configuration, when using the cylindrical gear or face gear split torque transmission system, it not only can improve the power-to-weight ratio of the helicopter main reducer, but also has the advantages of high reliability, weight loss and noise reduction, small error, and simple support structure [1-5].

For the power split transmission configuration, the load transmitted by both branches should be equal, or partial load will occur, or even the gear will be broken, which will affect the safety and service life of the drivetrain. Therefore, the load sharing performance is one of the important indicators of power split transmission configuration. In order to improve the load sharing performance of power split transmission, many scholars carry out extensive research on it. A gear web method with an elastic material that achieves equal load through greater damping of the elastic material and less torsional stiffness is proposed [6]. Some scholars studied the feasibility of using a balanced beam method for load sharing $[7,8]$. A clocking angle is defined as the design variable of the split path gearboxes [9]. The clocking angle can be adjusted to split a design load equally between two power paths. In addition, some scholars have also studied the influence of the flexible shaft on the dynamic load sharing properties [10-13].

\footnotetext{
* Corresponding author: meeghjin@nuaa.edu.cn
} 
Combined with the advantages of power split transmission with face-gear and cylindrical gear drive, the configuration of double split gear system with face gear and spur gear is put forward. Based on the concentrated mass method, a bending and torsion coupling dynamics model is established, and the influence of torsional stiffness of the compound gear shaft on the load sharing coefficient is studied emphatically, which provides a reference for improving the load sharing performance of the transmission system.

\section{Dynamic model of the transmission system}

Fig. 1 shows the face gear - spur gear power split transmission system structure diagram. For the convenience of narration, the first stage of the transmission system is defined as the input stage, the second stage is the split torque stage, and the third stage is the power confluence stage. In the figure, $Z_{i f}, Z_{i \mathrm{p}}, Z_{i j \mathrm{~s}}$ and $Z_{i j \mathrm{~h}}$ denote face gear, pinion and large gear of the split torque stage, pinion of the power confluence stage respectively $(i=R, L, j=1,2)$. Each transmission shaft is defined as follows: the shaft connecting the $Z_{\mathrm{Rf}}$ and $Z_{\mathrm{Rp}}$ is a right-end split torque shaft; the shaft connecting the $Z_{\mathrm{Lf}}$ and $Z_{\mathrm{Lp}}$ is a left-end split

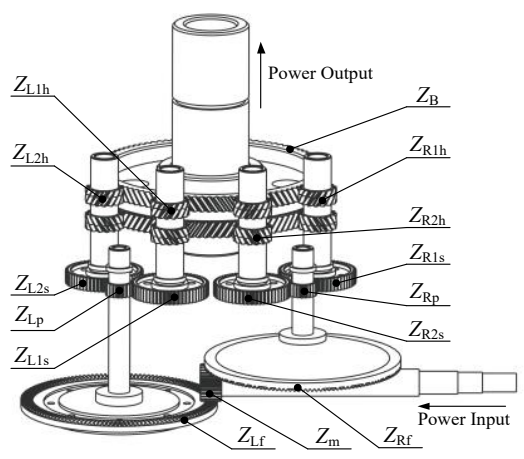

Fig.1. Sketch of the split-torque drive system torque shaft. The shafts connecting the $Z_{i j \mathrm{~s}}$ and $Z_{i j \mathrm{~h}}$ are compound gear shafts, from right to left, respectively called right-hand branch 1 , right-hand branch 2 , left-hand branch 1 and left-hand branch 2 .

Dynamic model of face gear - spur gear power split transmission system is shown in Fig. 2. In the model, the meshing stiffness of gear teeth, torsional stiffness of shaft and bearing support stiffness are represented by equivalent spring stiffness. Stiffness, damping, transmission error and clearance are indicated by the $K, c, e, b$ with the corresponding subscript, respectively. $K_{\text {inmf }}, K_{\text {inpjs }}$ and $K_{\text {inBjh }}$ denote the time-varying meshing stiffness of the corresponding gears, respectively, and the subscripts of meshing damping, transmission error and clearance of the gear pair are the same as the time-varying meshing stiffness. $K_{D m}$, $K_{\text {ifp }}, K_{i j s h}$ and $K_{B o}$ represent the torsional stiffness of input shaft, $i$-end split torque shaft, $i$ end compound gear shaft of branch $j$ and output shaft, respectively, and $c_{D m}, c_{i f p}$ and $c_{i j \mathrm{sh}}$ denote the torsional damping of the corresponding shaft respectively. $K_{m x}$ and $K_{m z}$ represent the support stiffness of the input shaft in the $X$ and $Z$ directions, respectively. $K_{i \mathrm{px}}, K_{i \mathrm{py}}$ and $K_{i \mathrm{pz}}$ represent the $X$-direction, $Y$-direction and axial support stiffness of the $i$-end split torque shaft respectively. $K_{i j \mathrm{x}}$ and $K_{i j \mathrm{y}}, K_{\mathrm{Bx}}$ and $K_{\mathrm{By}}$ represent the support stiffness of the compound gear shaft and output shafts in $X$ and $Y$ directions, respectively. The subscript of the support damping of the corresponding shafts is the same as the support stiffness.

The drive system has a total of 34 degrees of freedom, its generalized vector $\boldsymbol{Y}$ can be expressed as

$$
\begin{aligned}
\boldsymbol{Y}= & \left(\varphi_{\mathrm{D}}, \varphi_{\mathrm{m}}, \varphi_{\mathrm{Rf}}, \varphi_{\mathrm{Rp}}, \varphi_{\mathrm{R} 1 \mathrm{~s}}, \varphi_{\mathrm{R} 2 \mathrm{~s}}, \varphi_{\mathrm{R} 1 \mathrm{~h}}, \varphi_{\mathrm{R} 2 \mathrm{~h}}, \varphi_{\mathrm{Lf}}, \varphi_{\mathrm{Lp}}, \varphi_{\mathrm{L} 1 \mathrm{~s}}, \varphi_{\mathrm{L} 2 \mathrm{~s}}, \varphi_{\mathrm{L} 1 \mathrm{~h}}, \varphi_{\mathrm{L} 2 \mathrm{~h}}, \varphi_{\mathrm{B}}, \varphi_{\mathrm{o}},\right. \\
& \left.X_{\mathrm{nm}}, Z_{\mathrm{nm}}, X_{\mathrm{Rnp}}, Y_{\mathrm{Rnp}}, Z_{\mathrm{Rnp}}, X_{\mathrm{R} 1}, Y_{\mathrm{R} 1}, X_{\mathrm{R} 2}, Y_{\mathrm{R} 2}, X_{\mathrm{Lnp}}, Y_{\mathrm{Lnp}}, Z_{\mathrm{Lnp}}, X_{\mathrm{L} 1}, Y_{\mathrm{L} 1}, X_{\mathrm{L} 2}, Y_{\mathrm{L} 2}, X_{\mathrm{B}}, Y_{\mathrm{B}}\right)
\end{aligned}
$$

Where $\varphi_{\mathrm{D}}, \varphi_{\mathrm{m}}, \varphi_{\mathrm{B}}$ and $\varphi_{\mathrm{o}}$ are the torsional displacements of the input, $Z_{\mathrm{m}}, Z_{\mathrm{B}}$ and the output, respectively. $\varphi_{i f}, \varphi_{i \mathrm{p}}, \varphi_{i j \mathrm{~s}}$ and $\varphi_{i j \mathrm{~h}}$ are the torsional displacements of $Z_{i \mathrm{f}}, Z_{i \mathrm{p}}, Z_{i j \mathrm{~s}}$ and $Z_{i j \mathrm{~h}}$ respectively. $X_{\mathrm{nm}}$ and $Z_{\mathrm{nm}}$ are the lateral and axial displacement of the input shaft. $X_{\text {inp }}, Y_{\text {inp }}$ and $Z_{i n p}$ are the horizontal, vertical and axial displacements of the split torque shaft respectively. $X_{i j}$ and $Y_{i j}$ represent the horizontal displacement and the vertical displacement 
of the compound gear shafts respectively. $X_{\mathrm{B}}$ and $Y_{\mathrm{B}}$ are the horizontal and vertical displacements of the output shaft, respectively.

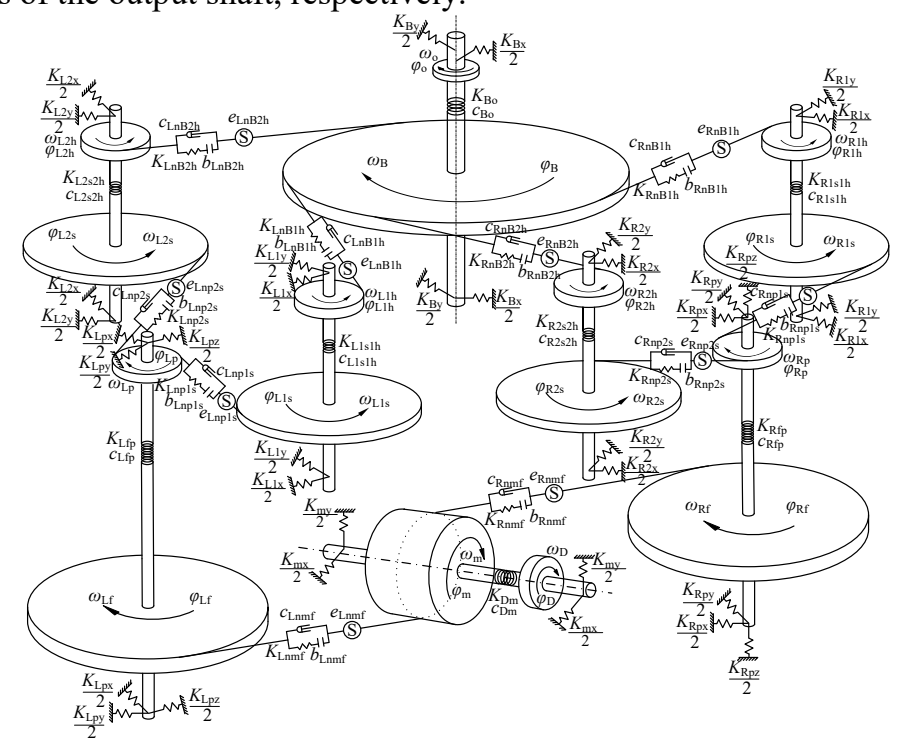

Fig.2. Dynamic model of twice split-torque drive system

\section{Differential equations of transmission system}

\subsection{Coordinate transformation equation}

Fig. 3 shows the local and generalized coordinates of the drive system. In the figure, $X$ and $Y$ represent the global coordinates, and $X^{*}$ and $Y^{*}$ represent the local coordinates. Among them, the direction of $Y$ or $Y^{*}$ coincides with the direction of the meshing line between the gear pairs. $\theta_{i \mathrm{p}}, \theta_{i j}, \theta_{i \mathrm{~B}}$ and $\theta_{\mathrm{B}}$ are the mounting angles between the respective gears.

According to the structural symmetrical relationship between the two input systems of split torque transmission, the transformation matrices between the generalized coordinates and the local coordinates can be obtained.

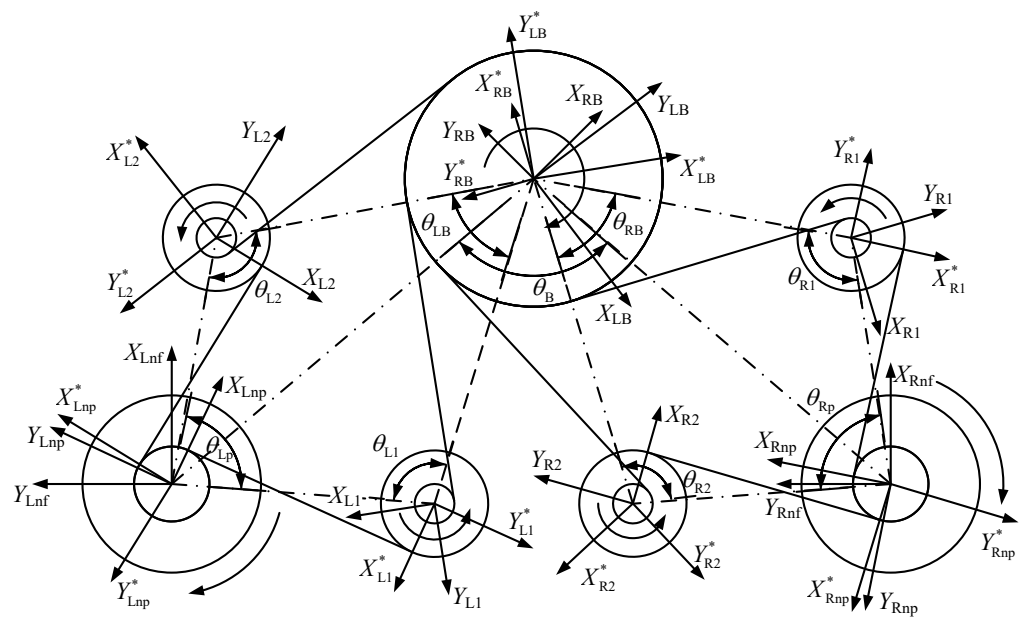

Fig.3. Relationship of partial and generalized coordinates of twice split-torque drive system 


\subsection{Nonlinear dynamic equations}

Let $F_{i n m f}, F_{\text {inpjs }}$ and $F_{i n \mathrm{Bjh}}$ respectively represent the meshing force between the gear pair $Z_{\mathrm{m}}$ and $Z_{i f}, Z_{i \mathrm{p}}$ and $Z_{i j \mathrm{~s}}, Z_{\mathrm{B}}$ and $Z_{i j \mathrm{~h} .}$. The meshing stiffness of face gear pair is calculated according to the literature calculation method $[14,15]$. The input torques are $T_{\mathrm{D}}$ and the load is $T_{\mathrm{o}}$. The concentrated masses of the gears $Z_{\mathrm{m}}, Z_{i \mathrm{f}}, Z_{i \mathrm{p}}, Z_{i j \mathrm{~s}}, Z_{i j \mathrm{~h}}$ and $Z_{\mathrm{B}}$ are expressed in $m_{\mathrm{m}}, m_{i f}, m_{i \mathrm{p}}, m_{i j \mathrm{~s}}, m_{i j \mathrm{~h}}$ and $m_{\mathrm{B}}$ respectively. The moments of inertia of the gears $Z_{\mathrm{m}}, Z_{i \mathrm{f}}, Z_{\mathrm{ip}}$, $Z_{i j \mathrm{~s}}, Z_{i j \mathrm{~h}}$ and $Z_{\mathrm{B}}$ are represented by $I_{\mathrm{m}}, I_{i \mathrm{f}}, I_{i \mathrm{p}}, I_{i j \mathrm{~s}}, I_{i j \mathrm{~h}}$ and $I_{\mathrm{B}}$, respectively. According to the system dynamics model and the force analysis, the nonlinear dynamic equation of the split torque drive system can be derived as

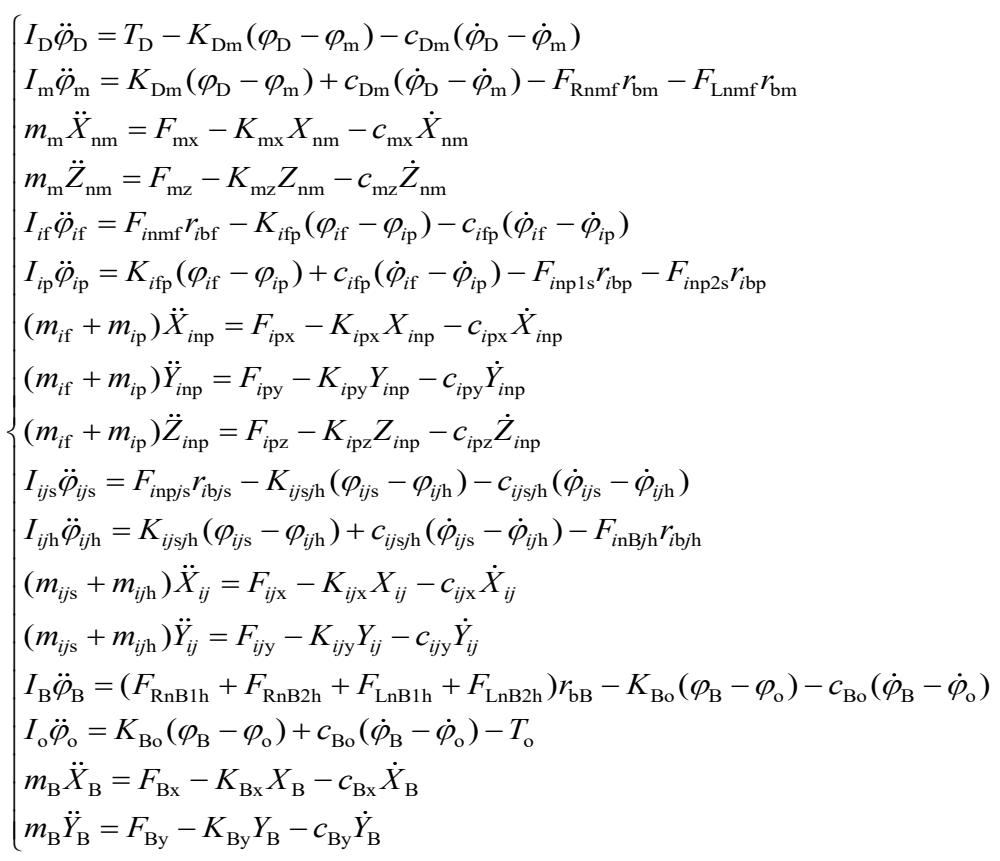

The displacement in Eq. (2) is unified as linear displacement, which can eliminate the rigid body displacement in the dynamic equations. In the nonlinear dynamic equations, the order of magnitude of each variable varies greatly, which affects the accuracy of the solution and the computational efficiency. Therefore, the kinetic equation is also dimensioned, and will not be repeated here.

\section{Analysis of load sharing coefficient}

\subsection{Analysis of load sharing coefficient of each branch}

Fig. 4 shows the load sharing coefficient of each branch of the powertrain as a function of time. From Fig.4 (a) to (e), it can be seen that the load sharing coefficient of two branches of each transmission stage is not equal. Among them, the maximum load factor of the input stage appears at the right end. The maximum load factor of the left and right split torque stages and the power confluence stages all appear in the branch 2 . When the system is in stable operation, the load sharing coefficient of each branch shows an approximate periodic variation. 


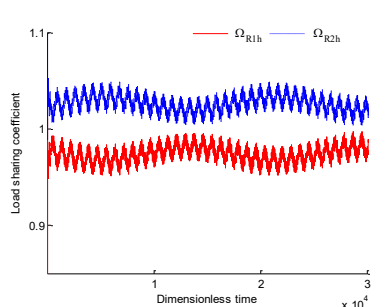

(a) Power input stage

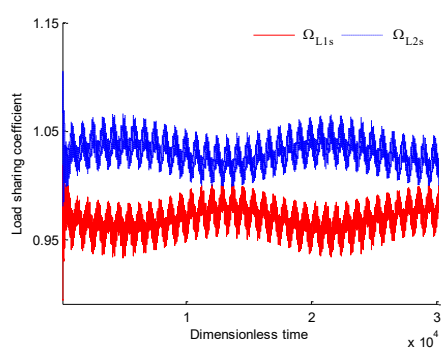

(d) Left side of split torque stage
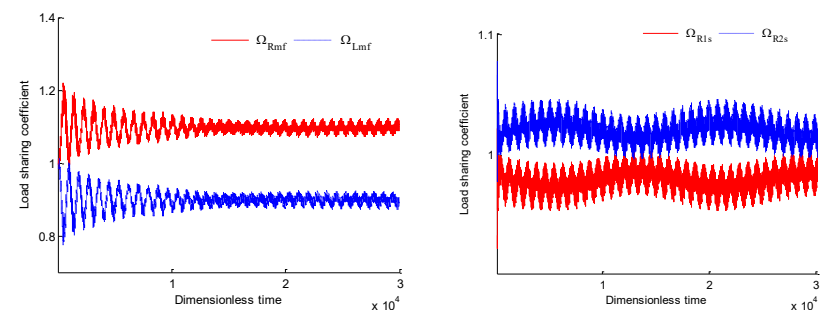

(c) Right side of power confluence stage

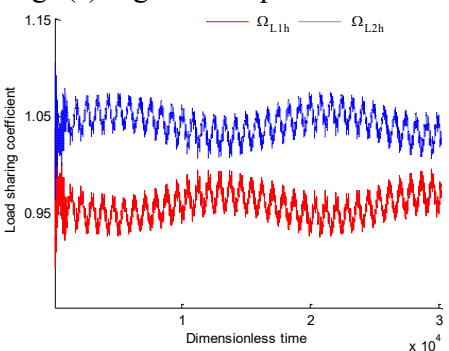

(e) Left side of power confluence stage

Fig. 4. Time history of load sharing coefficients of the system

\subsection{Effect of torsional stiffness of compound gear shafts on load sharing coefficient}

The influence of the torsional stiffness of the compound gear shaft on the load sharing performance of the transmission system is mainly studied. Fig. 5 shows the load sharing coefficient of each transmission stage with the torsional stiffness of the compound gear shaft. It can be seen from the figure that the change of torsional stiffness of the compound gear shaft has a significant effect on the load sharing coefficient of each transmission stage. The load sharing coefficient of the input stage decreases with the increase of the torsional stiffness of the compound shaft, but the load sharing coefficient of split torque stage and power confluence stage increases with the torsional stiffness of compound gear shaft.

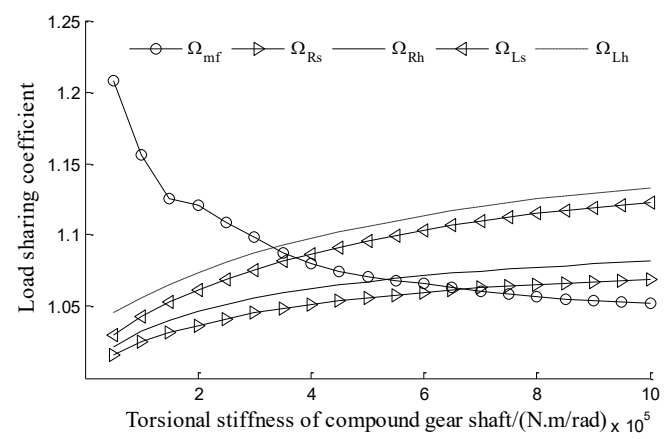

Fig.5. Impact of torsional stiffness of compound gear shaft on the load sharing coefficient

The geometric structure of the system has the symmetry. Taking the left branch of the split torque stage and the power confluence stage as an example, the meshing force between gear pairs is asymmetrical despite the geometric symmetry. Under the condition that the gear is loaded, the lateral micro-displacement of the gear center will lead to the difference of the micro-displacement of the gear torsion, resulting in the difference of the torques to the left and right branches of the split torque stage. With the increase of the torsional stiffness of the compound gear shaft, the load sharing coefficient of the split torque stage and the power confluence stage is less sensitive to it. The main reason is that with the 
increase of torsional stiffness, the proportion of gear torsion angle caused by the lateral micro-displacement of gear center is gradually increased, and the proportion of torsional deformation decreases gradually. When the torsional stiffness of the compound gear shaft increases, the flexibility of the split shaft increases relatively, so the deformation coordination ability increases, and the load performance of the input stage is improved.

\section{Conclusion}

The torsional stiffness of the compound gear shaft has a great influence on the load sharing coefficient of input stage, split torque stage and power confluence stage. Reducing the torsional stiffness of the compound gear shaft can improve the load sharing performance of the split torque stage and the power confluence stage. However, the input stage load sharing performance degrades as the torsional stiffness of the compound gear shaft decreases. Therefore, in order to obtain a better dynamic load-sharing performance, the torsional stiffness of the transmission shaft should be properly configured.

This work supported by Research Program supported by the National Natural Science Foundation (Grant No. 51475226), China.

\section{References}

[1] G. White. P. I. Mech. Eng. C-J. Mec., 188, 281-288 (1974)

[2] G. White. P. I. Mech. Eng. G-J. Aerosp. Eng., 212, 117-123 (1998).

[3] A. Segade-Robleda, José-Antonio, M. López-Lago. Mech. Eng. InTech, 4, 55-74( 2012).

[4] G. F. Heath, B. Jr. Bossler. NASA C. R. 191057, (1993)

[5] R. E. Handschuh, D. G. Lewicki, G. F. Heath. NASA Tech. Memo. 107227, (1996)

[6] J. G. Kish. NASA C. R. 191079, 1-212 (1993)

[7] M. Rashidi, T. Krantz. NASA Tech. Memo. 105681, 1-12 (1992)

[8] T. L. Krantz. NASA Tech. Memo. 106410, 1-43 (1994)

[9] T. L. Krantz, I. R. Delgado. NASA Tech. Memo. 107202, 1-11 (1996)

[10]G. White. P. I. Mech. Eng. G-J. Aerosp. Eng, 212, 117-123 (1998)

[11]Y. Gmirya, S. He, G. Buzel. the American Helicopter Society 65th Annual Forum, 977-986 (2009)

[12] Y. Gmirya, M. Alulis, P. Palcic, L. Leigh. the American Helicopter Society 67th Annual Forum, 2323-2334 (2011)

[13] Y. F. Gui, R. P. Zhu, B. B. Fu. J. Aerosp. P., 29, 2265-2268 (2014)

[14]Z. Hu, J. Tang, S. Chen. ASME J. Mech. Design., 135, 1-7 (2013)

[15]G. H. Jin. Research on strength and dynamic characteristics of orthogonal face gear drives. NUAA, (2012) 